\title{
CORRELATION FUNCTIONS OF THE ISING MODEL WITH MULTISITE INTERACTION ON THE HUSIMI LATTICE
}

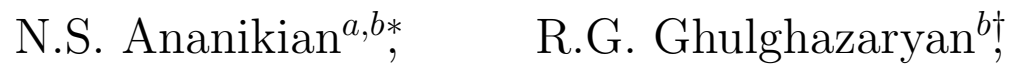 \\ N.Sh. Izmailian ${ }^{b, c \ddagger}$ \\ ${ }^{a}$ Department of Physics and Centre for Nonlinear Studies, \\ Hong Kong Baptist University, Hong Kong, China \\ ${ }^{b}$ Department of Theoretical Physics, Yerevan Physics Institute, \\ Alikhanian Br.2, 375036 Yerevan, Armenia \\ ${ }^{c}$ Institute of Physics, Academia Sinica, Taipei 11529, Taiwan
}

October 11, 2018

\begin{abstract}
We consider a general spin-1/2 Ising model with multisite interaction on the Husimi lattice with the coordination number $q$ and derive an analytical expression of correlation functions for stable fixed points of the corresponding recurrence relation. We show that for $q=2$ our model transforms to the two-state vertex model on the Bethe lattice with $q=3$ and for the case $q=3$, with only nearest neighbour interactions, we transform our model to the corresponding model on the Bethe lattice with $q=3$, using the Yang-Baxter equations.
\end{abstract}

*e-mail: ananik@jerewan1.yerphi.am

${ }^{\dagger}$ e-mail: ghulr@moon.yerphi.am

${ }^{\ddagger}$ e-mail: izmailan@phys.sinica.edu.tw 


\section{Introduction}

Correlation functions play an important role in the understanding of the critical behavior of the statistical mechanical models. There were received some exact results for correlation functions and critical exponents for the Euclidean plane models using conformal invariance [1, 2]. Usually Bethe-like (Husimi) lattices considered as embedded in the infinite-dimensional Euclidean space. However Bethe-like lattices can be embedded in a two-dimensional space of constant negative curvature (the hyperbolic plane) with fixed bond angles and lengths. These hierarchical structures like the Bethe and Husimi lattices can be described as a conformal (regular) tiling of the hyperbolic plane [10, 11]. It is believed that several among its interesting thermal properties could persist for regular lattices, for which the exact calculation is so far intractable.

The exact correlation function for Ising model on the Bethe lattice was obtained by Mukamel [6] and Falk []] and for Potts model on the Cayley tree in zero magnetic field by Wang and $\mathrm{Wu}[8]$. The two-vertex model on the Bethe lattice with the coordination number $q=3$ was explored [3], a closed formula for correlation functions in stable fixed points of the corresponding recurrence relation was derived and two types of first-order phase transitions which were distinguished according to the behavior of correlation functions in the high-temperature phase were obtained [3]. Recently Izmailian and Hu derived the exact correlation functions for the Ising, BEG and the most general spin $-S$ models on the Bethe lattice [1, 5]. Applying combinatorial approach and graph expansion Yang and $\mathrm{Xu}$ calculated multi-spin correlation function of the Ising model on Bethe-type lattice [9], they results indicates no existence of long range correlation at any finite temperature which implies that no finite temperature phase transition occurs. Recently, the multisite interaction Ising model on the Husimi lattice was investigated. First, it was shown that this approach yields good approximation for the ferromagnetic phase diagrams [14] and closely matches the exact results obtained on a Kagome lattice [16]. The reason for studying the Ising model with multisite interaction is that it plays an important role in the investigations of real physical systems such as binary alloys [17], classical fluids 18], solid ${ }^{3} \mathrm{He}$ [19], liquid bilayers [20], and rare gases [21].

In this paper we derive a closed formula for correlation functions of the multisite interaction Ising model on the Husimi lattice for arbitrary coordination number $q$ and show that our model for the case $q=2$ coincides with the two-vertex model [3] and for only nn (nearest neighbour) interaction Ising model it transforms into the corresponding model on the Bethe lattice with $q=3$.

The plan of the paper is as follows. In section 2, we define the model, derive the relevant recurrence relation and magnetization deep within the tree. In section 3, we derive a closed formula for correlation functions $g(n)=\left\langle S_{1} S_{n}\right\rangle-\left\langle S_{1}\right\rangle\left\langle S_{n}\right\rangle$, where the symbol $\langle\ldots\rangle$ signifies the mean value and $S_{1}$ and $S_{n}$ are spins on 0th and $(n-1)$ th shells respectively. Section 4 is devoted to the star-triangle relation between the partition functions of Ising models on the Husimi and Bethe lattices.

\section{Recurrence relation and magnetization}

The pure Husimi lattice [12, 13], shown in Fig.1, is characterized by the coordination number $q$, the number of triangles which goes out from each site, the 0th-generation is a 
single central triangle.

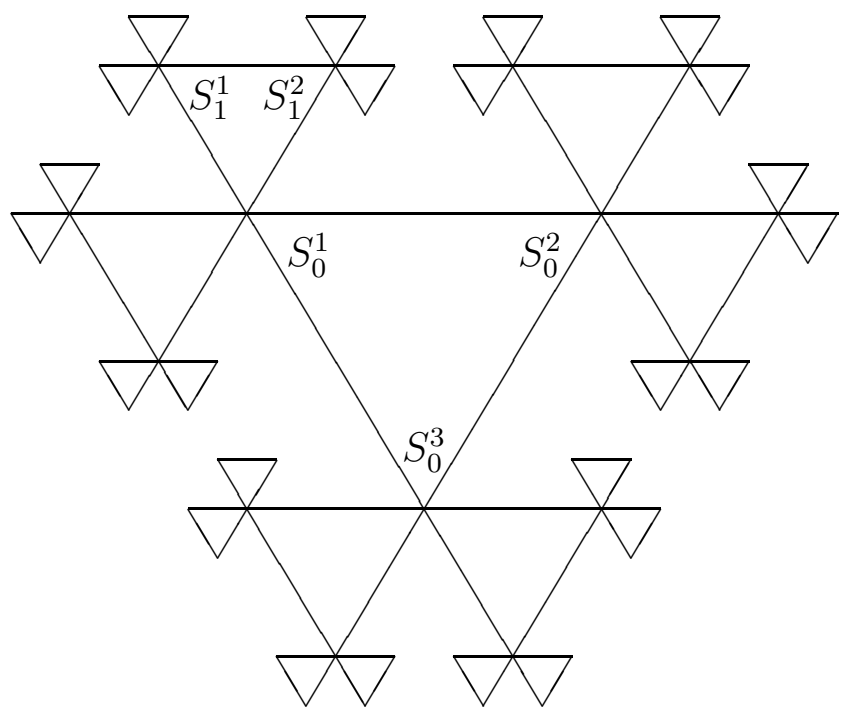

Fig.1 The Husimi lattice with $q=3$.

The general Hamiltonian with pair, three-site interactions in an external magnetic field is

$$
-\frac{H}{k T}=J_{2} \sum_{\langle i j\rangle} S_{i} S_{j}+J_{3} \sum_{\triangle} S_{i} S_{j} S_{k}+h \sum_{i} S_{i},
$$

where $S_{i}$ takes values \pm 1 ; the first sum goes over all nearest neighbour pairs of the lattice sites, the second sum goes over all triangular faces of the Husimi lattice and the third one goes over all sites. The advantage of the Husimi lattice introduced here is that for the models formulated on it, the exact recurrence relation can be derived. From Fig.1 it is apparent that if the graph is cut at the central triangle, then it splits up into three identical disconnected pieces and each of them contains $\gamma$ sub-lattices, where we defined $\gamma=q-1$. Then the partition function

$$
Z(N)=\sum_{\{S\}} \exp \left[-\frac{H}{k T}\right]
$$

may be written

$$
\begin{aligned}
Z(N)= & \sum_{\left\{S_{0}\right\}} \exp \left[J_{2}\left(S_{0}^{1} S_{0}^{2}+S_{0}^{1} S_{0}^{3}+S_{0}^{2} S_{0}^{3}\right)+J_{3} S_{0}^{1} S_{0}^{2} S_{0}^{3}+\frac{h}{q}\left(S_{0}^{1}+S_{0}^{2}+S_{0}^{3}\right)\right] \times \\
& \times B^{\gamma}\left(N, S_{0}^{1}\right) B^{\gamma}\left(N, S_{0}^{2}\right) B^{\gamma}\left(N, S_{0}^{3}\right),
\end{aligned}
$$

where $S_{0}^{i}$ are spins of the central triangle and $B(N, S)$ is the partition function of one sub-lattice containing $N$ shells and with the root-spin fixed in the state $S$. Here we use the same notations as in Kolesik paper [3]. Because of our Husimi lattice is constructed from triangles we can introduce statistical weights depending on three spins located on triangle's vertexes. Introducing the notations

$$
w\left(S_{1}, S_{2}, S_{3}\right)=\exp \left[J_{2}\left(S_{1} S_{2}+S_{1} S_{3}+S_{2} S_{3}\right)+J_{3} S_{1} S_{2} S_{3}+\frac{h}{q}\left(S_{1}+S_{2}+S_{3}\right)\right]
$$


and

$$
\begin{aligned}
& a_{0}=w(-,-,-)=\exp \left[3 J_{2}-J_{3}-3 \frac{h}{q}\right] \\
& a_{1}=w(+,-,-)=w(-,+,-)=w(-,-,+)=\exp \left[-J_{2}+J_{3}-\frac{h}{q}\right] \\
& a_{2}=w(+,-,+)=w(+,+,-)=w(-,+,+)=\exp \left[-J_{2}-J_{3}+\frac{h}{q}\right] \\
& a_{3}=w(+,+,+)=\exp \left[3 J_{2}+J_{3}+3 \frac{h}{q}\right]
\end{aligned}
$$

we can present the partition function as follows

$$
Z(N)=\sum_{S_{0}^{1}, S_{0}^{2}, S_{0}^{3}} w\left(S_{0}^{1}, S_{0}^{2}, S_{0}^{3}\right) B^{\gamma}\left(N, S_{0}^{1}\right) B^{\gamma}\left(N, S_{0}^{2}\right) B^{\gamma}\left(N, S_{0}^{3}\right)
$$

Using the "cutting apart" procedure described above, one can derive the recurrence relation for $B(N, S)$

$$
B(N, S)=\sum_{S_{1}, S_{2}} w\left(S, S_{1}, S_{2}\right) B^{\gamma}\left(N-1, S_{1}\right) B^{\gamma}\left(N-1, S_{2}\right) .
$$

The magnetization of the central site we can express as follows

$$
m=\left\langle S_{0}^{1}\right\rangle=(Z(N))^{-1} \sum_{S_{0}^{1}, S_{0}^{2}, S_{0}^{3}} S_{0}^{1} w\left(S_{0}^{1}, S_{0}^{2}, S_{0}^{3}\right) B^{\gamma}\left(N, S_{0}^{1}\right) B^{\gamma}\left(N, S_{0}^{2}\right) B^{\gamma}\left(N, S_{0}^{3}\right) .
$$

Let the following variables be introduced

$$
q_{N}(S)=\frac{B(N, S)}{B(N,+)} \quad \text { and } \quad x_{N}=\frac{B(N,-)}{B(N,+)}
$$

Then using (9) one can write the recurrence relation for $x_{N}$

$$
x_{N}=f\left(x_{N-1}\right), \quad f(x)=\frac{a_{2}+2 a_{1} x^{\gamma}+a_{0} x^{2 \gamma}}{a_{3}+2 a_{2} x^{\gamma}+a_{1} x^{2 \gamma}} .
$$

The $x_{N}$ has no direct physical meaning, but through it one can express the magnetization of the central site

$$
m=\frac{a_{3}+a_{2} x_{N}^{\gamma}-a_{1} x_{N}^{2 \gamma}-a_{0} x_{N}^{3 \gamma}}{a_{3}+3 a_{2} x_{N}^{\gamma}+3 a_{1} x_{N}^{2 \gamma}+a_{0} x_{N}^{3 \gamma}}
$$

and other thermodynamic parameters, since we can say that the $x_{N}$ determine the states of the system.

In this paper we restrict our treatment to ferromagnetic case $\left(J_{2}>0\right.$ and $\left.J_{3}>0\right)$ and antiferromagnetic one $\left(J_{3}<0\right.$ or/and $\left.J_{2}<0\right)$ at high temperatures when our one dimensional mapping (11) has stable fixed points only. For the antiferromagnetic case at low temperatures $T<T^{*}$ the single fixed point $x_{0}$ become unstable when

$$
\left.\frac{\partial}{\partial x} f(x)\right|_{x=x_{0}}<-1
$$


and a so-called period doubling bifurcation occurs: the recursive sequence $\left\{x_{N}\right\}$ converges now not to the single fixed point but to the stable two-cycle $\left\{x_{1}, x_{2}\right\}$. This phase should be explained as an arising of a two-sublattice phase such that $x_{1}$ and $x_{2}$ determine the states on each sublattice [12, 13, 14]. In terms of one dimensional maps the condition of the second order phase transition point of ferromagnetic models is

$$
\left.\frac{\partial}{\partial x} f(x)\right|_{x=x_{c}}=1
$$

and the second order phase transition point of antiferromagnetic models [15] is

$$
\left.\frac{\partial}{\partial x} f(x)\right|_{x=x_{c}}=-1
$$

We restrict our treatment to stable fixed points of the mapping (11) because the procedure applied later usable for fixed points only (see Sect. 3). The fixed point $x$ of the recurrence relation (11) is a solution of the equation

$$
x=f(x) \quad \text { or } \quad a_{1} x^{2 \gamma+1}-a_{0} x^{2 \gamma}+2 a_{2} x^{\gamma+1}-2 a_{1} x^{\gamma}+a_{3} x-a_{2}=0 .
$$

Thus, for the stable fixed point $x$ one obtains for the magnetization

$$
m=\lim _{N \rightarrow \infty} \frac{1-x_{N}^{\gamma} x_{N+1}}{1+x_{N}^{\gamma} x_{N+1}}=\frac{1-x^{q}}{1+x^{q}} .
$$

\section{Correlation Functions}

One can define correlation functions on the Husimi lattice as follows

$$
g(n)=\left\langle S_{1} S_{n}\right\rangle-\left\langle S_{1}\right\rangle\left\langle S_{n}\right\rangle
$$

where spin $S_{1}$ belongs to the central triangle (0th shell) and $S_{n}$ located somewhere in the $(n-1)$ th shell. Using denotation (10) and recurrence relation (9) we can express $\left\langle S_{1} S_{n}\right\rangle$ as follows

$$
\begin{aligned}
\left\langle S_{1} S_{n}\right\rangle= & \lim _{N \rightarrow \infty} \frac{\sum S_{1} q_{N}^{\gamma}\left(S_{1}\right) w\left(S_{1}, y_{1}, z_{1}\right) q_{N}^{\gamma}\left(y_{1}\right) q_{N}^{\gamma-1}\left(z_{1}\right) w\left(z_{1}, y_{2}, z_{2}\right) q_{N-1}^{\gamma}\left(y_{2}\right) \cdots}{\sum q_{N}^{\gamma}\left(S_{1}\right) w\left(S_{1}, y_{1}, z_{1}\right) q_{N}^{\gamma}\left(y_{1}\right) q_{N}^{\gamma-1}\left(z_{1}\right) w\left(z_{1}, y_{2}, z_{2}\right) q_{N-1}^{\gamma}\left(y_{2}\right) \cdots} \\
& \frac{\cdots q_{N-n+2}^{\gamma-1}\left(z_{n-1}\right) w\left(z_{n-1}, y_{n}, S_{n}\right) q_{N-n+1}^{\gamma}\left(y_{n}\right) q_{N-n+1}^{\gamma}\left(S_{n}\right) S_{n}}{\cdots q_{N-n+2}^{\gamma-1}\left(z_{n-1}\right) w\left(z_{n-1}, y_{n}, S_{n}\right) q_{N-n+1}^{\gamma}\left(y_{n}\right) q_{N-n+1}^{\gamma}\left(S_{n}\right)} .
\end{aligned}
$$

Let us define following matrices with elements

$$
\begin{aligned}
M\left(z_{i}, z_{i+1}\right) & =\sum_{y_{i+1}} q_{N-i+1}^{\gamma-1}\left(z_{i}\right) w\left(z_{i}, y_{i+1}, z_{i+1}\right) q_{N-i}^{\gamma}\left(y_{i+1}\right) \\
A^{\prime}\left(S_{n}, S_{1}\right) & =S_{n} S_{1} q_{N}\left(S_{1}\right) q_{N-n+1}^{\gamma}\left(S_{n}\right) \\
A\left(S_{n}, S_{1}\right) & =q_{N}\left(S_{1}\right) q_{N-n+1}^{\gamma}\left(S_{n}\right) .
\end{aligned}
$$

Note that for stable fixed points of the mapping (11) the difference between $q_{N}(S)$ and $q_{N-i}(S)$ disappears for $N \rightarrow \infty$ and for all finite values $i$. Hence, for stable fixed points 
labeling rows (columns) of these matrices as,+- from left to right (from up to down), by (16), (17) and (18) we have

$$
\begin{aligned}
M & =\left(\begin{array}{cc}
a_{3}+a_{2} x^{\gamma} & a_{2}+a_{1} x^{\gamma} \\
x^{\gamma-1}\left(a_{2}+a_{1} x^{\gamma}\right) & x^{\gamma-1}\left(a_{1}+a_{0} x^{\gamma}\right)
\end{array}\right) ; \\
A & =\left(\begin{array}{cc}
1 & x \\
x^{\gamma} & x^{\gamma+1}
\end{array}\right) \quad \text { and } \quad A^{\prime}=\left(\begin{array}{cc}
1 & -x \\
-x^{\gamma} & x^{\gamma+1}
\end{array}\right) .
\end{aligned}
$$

Using (16) - (20) we can rewrite (15) as follows

$$
\left\langle S_{1} S_{n}\right\rangle=\frac{\operatorname{Tr}\left(A^{\prime} M^{n}\right)}{\operatorname{Tr}\left(A M^{n}\right)} .
$$

Using (13) it is easy to show that matrices $M$ (19) and $A(20)$ commute. Thus, they can be diagonalized simultaneously. After performing this diagonalization we have

$$
\left\langle S_{1} S_{n}\right\rangle=\left(\frac{1-x^{q}}{1+x^{q}}\right)^{2}+\frac{4 x^{q}}{\left(1+x^{q}\right)^{2}}\left(\frac{\lambda_{2}(x)}{\lambda_{1}(x)}\right)^{n}
$$

where

$$
\begin{aligned}
& \lambda_{1}(x)=a_{3}+2 a_{2} x^{\gamma}+a_{1} x^{2 \gamma}, \\
& \lambda_{2}(x)=x^{\gamma-1}\left(a_{0} x^{\gamma}-a_{2} x+a_{1}\left(1-x^{\gamma+1}\right)\right)
\end{aligned}
$$

are the eigenvalues of matrix $M$. Note that, for stable fixed points $\left\langle S_{1}\right\rangle=\left\langle S_{n}\right\rangle=m$. Thus, using formula (14) for $m$ we can reveal the following formula for correlation functions

$$
g(n)=\frac{4 x^{q}}{\left(1+x^{q}\right)^{2}}\left(\frac{\lambda_{2}(x)}{\lambda_{1}(x)}\right)^{n}
$$

where $\lambda_{1}(x)$ and $\lambda_{2}(x)$ are defined in (23), (24).

One can prove easily that the correlation function decreases exponentially with the distance $n$ independently of $x$, which is a consequence of the infinite dimension of the Husimi lattice.

For a period doubling bifurcation of the mapping (11) the matrices corresponding to the $M$ and $A$ matrices and depending on the $x_{1}$ and $x_{2}$ points of the two cycle $\left\{x_{1}, x_{2}\right\}$ do not commute, so the procedure applied above is unusable for this case.

\section{Connection with the two-vertex and nn interaction Ising models on the Bethe lattice}

In the previous section we have derived a closed formula for correlation functions on the Husimi lattice. Now we shall show that for $q=2$ our model coincides with the two-vertex model on the Bethe lattice with $q=3$. First we recall the definition of the model. Let us consider the Bethe lattice with $N$ shells and the coordination number $q=3$. The central point $O$ and the adjacent edges are considered as the 0th shell. Each edge, including the free ends on the surface of the tree, can be in one of the two distinct spin states $S \in\{+,-\}$. To each vertex one ascribe the statistical weight $w\left(S_{1}, S_{2}, S_{3}\right)$ depending on the number of incident edges in the $(+)$-state. Let $a_{i}$ denote the corresponding statistical weight of the vertex with $i$ incident edges in the state + . Using notation (3) and Fig. 2 it is easy to see now that our models coincide. 


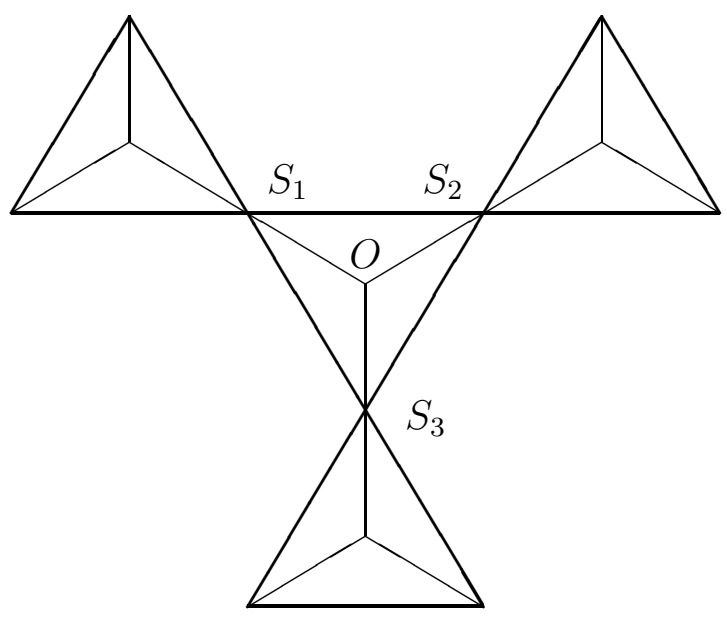

Fig.2 The Husimi and Bethe lattices with $q=2$ and $q=3$ respectively.

In the following we shall show that our model with only nn interaction $\left(J_{3}=h=\right.$ 0 ) and $q=3$ transforms to the nn interaction Ising model on the Bethe lattice with $q=3$. In this case we shall use widely known star-triangle transformation (or the Yang -Baxter equations) [1] to transfer our model to the Bethe lattice one. The star-triangle transformation replaces a star consisting of three spins interacting with a central spin, by a triangle of spins interacting with one another and vice versa (Fig.3).

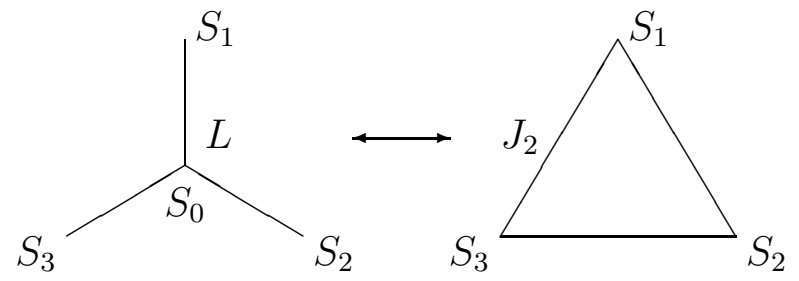

Fig.3 The star-triangle transformation.

Let us consider the simplest case in which the interactions of all directions are equal. Then summing over the central spin in the partition function of a star and equaling it to the partition function of a triangle with some coefficient $R$, we have

$$
\sum_{S_{0}} \exp L\left(S_{0} S_{1}+S_{0} S_{2}+S_{0} S_{3}\right)=R \exp J_{2}\left(S_{1} S_{2}+S_{1} S_{3}+S_{2} S_{3}\right)
$$

where $L$ and $J_{2}$ are the pair interaction parameters of the Bethe and Husimi lattices respectively. After performing the summation over $S_{0}$ and substituting $S_{i}= \pm 1, i=1,2,3$ we obtain the Yang-Baxter equations in the form

$$
\begin{aligned}
2 \cosh (3 L) & =R \exp \left(3 J_{2}\right), \\
2 \cosh (L) & =R \exp \left(-J_{2}\right),
\end{aligned}
$$

From notation (3) it's follows

$$
a_{0}=a_{3}=\exp \left(3 J_{2}\right) \quad \text { and } \quad a_{1}=a_{2}=\exp \left(-J_{2}\right) .
$$


Due to summation over spins located inside of triangles in star-triangular transformation one can obtain

$$
g_{H}(n)=g_{B}(2 n)
$$

where $g_{H}(n)$ and $g_{B}(2 n)$ are the correlation functions of the Husimi and Bethe lattices respectively. Thus, for correlation functions of the nn interaction Ising model on the Bethe lattice we have

$$
g_{B}(n)=\frac{4 x^{3}}{\left(1+x^{3}\right)^{2}} r^{n}(x)
$$

where

$$
r(x)=\frac{x(\exp (2 L)-x)}{x^{2}+\exp (2 L)} .
$$

Here we used the fact that $x$ is a stable fixed point of the corresponding recurrence relation on the Bethe lattice and it coincides with Husimi's lattice one. Here also one can prove that, independently of $x$, the correlation function decreases exponentially with the distance $n$, which is a consequence of the infinite dimension of the Bethe lattice.

From (26), (27) after simple algebra one can obtain

$$
\sinh 2 L \sinh 2 J_{2}=k^{-1}
$$

where

$$
k^{-1}=\frac{\sinh ^{3} 2 L}{2\left(\cosh 3 L \cosh ^{3} L\right)^{\frac{1}{2}}} .
$$

First the star-triangle transformation was used for triangle and honeycomb lattices [1]. It was obtained that for triangle and honeycomb lattices the condition of the critical point is $k=1$. For the ferromagnetic Ising model on the Bethe lattice it is well known that the critical point is

$$
L_{c}=\frac{1}{2} \ln \frac{q}{q-2}
$$

where $q$ is the coordination number of the lattice. From (30) follows that for the case $q=3$ parameter $k$ is unequal to $1\left(k=\frac{3 \sqrt{14}}{8}\right)$. From (29) one can obtain the critical point of the ferromagnetic Ising model on the Husimi lattice $\left(J_{2}\right)_{c}=\frac{1}{4} \ln \frac{7}{3}$ (the last result can be derived in a straightforward manner, see Sect. 2).

\section{Concluding remarks}

In this paper we have derived a closed formula for correlation functions of the multisite interaction Ising model in the presence of an external magnetic field. We showed that the correlation function decreases exponentially with the distance which indicates no existence of long range correlation at any finite temperature this is a consequence of the infinite dimension of the Husimi lattice in Euclidean space. This result is in good agreement with the results obtained in papers [3, 9]. All our obtained formulae contain only statistical weights $a_{i}$, which depend on the spins located in the vertexes of triangles. Hence, our formulae can be used also for other types of Hamiltonians where nn (nearest neighbour) and three-site interactions are present, for example Potts model, etc. 
The star-triangle relation (the Yang-Baxter equations) maps a high-temperature model on the Bethe lattice with $q=3$ to the high-temperature model on the Husimi cactus. Together with nonanalytic behavior of the recurrence relations of the Bethe and Husimi lattices one can use them to obtain the critical exponents and correlation functions too. In conclusion, let us note that the results deduced on the Husimi and Bethe-like lattices are in good agreement with results obtained on planar lattices, and according to Gujrati, Bethe or Bethe-like (Husimi cactus) calculations are more reliable than conventional mean-field calculations [22].

We are grateful to Professors A. Belavin, R. Flume, B. Hu and M. Roger for useful discussions. We would like to thank Professor J. Monroe for calling our attention to Ref. [7].

This work was partly supported by the Grant INTAS-96-690 and the grants from the Hong Kong Research Grants Council and the Hong Kong Baptist University. One of us (N. Sh. I.) thanks the National Science Council of the Republic of China (Taiwan) for financial support under grant No. NSC 87-2112-M-001-046. 


\section{References}

[1] R. J. Baxter, Exactly Solved Models in Statistical Mechanics (Academic Press, London, 1981).

[2] J. L. Cardy, In "Phase Transitions and Critical Phenomena", Vol. 11 (C. Domb and J. L. Lebowitz), Ch. 2. (Academic Press, London, 1987).

[3] M. Kolesik, Int. J. Mod. Phys. B6, 3469 (1992).

[4] Chin-Kun Hu and N. Sh. Izmailian, Phys. Rev. E 58 (1998), in press.

[5] N. Sh. Izmailian and Chin-Kun Hu, Physica A 254, 198 (1998).

[6] D. Mukamel, Phys. Lett. A50, 339 (1974).

[7] H. Falk, Phys. Rev. B12, 5184 (1974).

[8] Y. K. Wang, F. Y. Wu, J. Phys. A9, 593 (1976).

[9] Z.R. Yang, Chang Ye Xu, Commun. Theor. Phys. Vol. 22, 419-424 (1994).

[10] R. Mosseri and J. F. Sadoc, J. Phys. France Lett. 43, L249 (1982).

[11] J. A. de Miranda-Neto and Fernando Moraes, J. Phys. I France 3, 29 (1993).

[12] N. S. Ananikian et. al, Fractals, Vol. 5, 175 (1997).

[13] N. S. Ananikian and S.K.Dallakian, Physica D107, 75 (1997).

[14] J. L. Monroe, J. Stat. Physics, 65, 255 (1991).

[15] N. S. Ananikian, A. Z. Akheyan, Phys. Lett. A 186, 171 (1994).

[16] X. N. Wu and F. Y. Wu, J. Phys. A : Math. Gen. 22, L1031 (1989).

[17] D. F. Styer, M. K. Phani and J. I. Lebowitz, Phys. Rev. B34, 3361 (1986).

[18] M. Grimsditch, P. Loubeyre and A. Polian, Phys. Rev. B33, 7192 (1986).

[19] M. Roger, J. H. Hetherington and J. M. Delrieu, Rev. Mod. Phys. 55, 1 (1983).

[20] H. L. Scott, Phys. Rev. A37, 263 (1988).

[21] J. A. Barker, Phys. Rev. Lett. 57, 230 (1986).

[22] P. D. Gujrati, Phys. Rev. Lett. 74, 809 (1995). 